\title{
Phenotypic and Kinetic Changes of Myeloid Lineage Cells in Innate Response to Chikungunya Infection in Cynomolgus Macaques
}

Brandon J. Beddingfield $^{1 *}$, Chie Sugimoto ${ }^{4 *}$, Marcelo J. Kuroda ${ }^{5}$, Eryu Wang ${ }^{6}$, Scott C. Weaver ${ }^{6,7}$, Kasi E. Russell-Lodrigue ${ }^{2}$, Stephanie Z. Killeen ${ }^{1}$, Chad J. Roy ${ }^{1,3 * *}$

Divisions of Microbiology $y^{1}$ and Veterinary Medicine ${ }^{2}$, Tulane National Primate Research Center, Covington, LA, USA

${ }^{3}$ Department of Microbiology and Immunology, Tulane University School of Medicine, New Orleans, LA, 70112, USA

${ }^{4}$ Division of Host Defense, Institute for Frontier Medicine, Dokkyo Medical University, Mibu, Tochigi, Japan

${ }^{5}$ Center for Immunology and Infectious Diseases, and California National Primate Research Center, University of California, Davis, California, USA.

${ }^{6}$ Department of Microbiology \& Immunology, University of Texas Medical Branch, Galveston, TX, USA

${ }^{7}$ World Reference Center for Emerging Viruses and Arboviruses, Institute for Human Infections and Immunity, University of Texas Medical Branch, Galveston, TX, USA.

*co-first authors

**correspondence: C.J.R. (croy@tulane.edu) 


\begin{abstract}
Chikungunya is an emerging threat, spreading across multiple continents. The immune response to infection can lead to protection and convalescence, or result in long term sequelae such as arthritis. Early innate immune events during acute infection have been characterized for some cell types, but more must be elucidated with respect to cellular responses of monocytes and other myeloid lineage cells. In addition to their roles in protection and inflammation resolution, monocytes and macrophages are sites for viral replication and reservoirs for the virus. They are also found in joints post-infection, possibly playing a role in long term CHIKV-induced pathology. We examined kinetic and phenotypic changes in myeloid lineage cells, including monocytes, in cynomolgus macaques early after infection with CHIKV. We found increased turnover of monocytes and mDCs early during infection, with an accompanying decreases in both cell types, as well as a simultaneous increase in pDC number. An increase in CD16 and CD14 was seen along with a decrease in monocyte HLA-DR expression within 3 days of infection, potentially indicating monocyte deactivation. A transient decrease in T cells, B cells and NK cells correlates with lymphocytopenia observed during human infections with CHIKV. CD4+ T cell turnover decreased in blood, indicating relocation of cells to effector sites. This data indicates CHIKV influences turnover rates and kinetics of myeloid lineage cells early during infection, and may prove useful in development of therapeutics and evaluation of infection-induced pathogenesis.
\end{abstract}

\title{
Introduction
}

Chikungunya is a rapidly emerging arboviral disease, with outbreaks occurring commonly in India and Southeast Asia ${ }^{1}$, that has resulted in approximately 1.9 million cases worldwide ${ }^{2}$. Since at least 2013, autochthonous transmission has occurred in the Americas ${ }^{3}$, with over 992,000 cases reported, including 332,000 in the United States and Canada (https://www.paho.org/data/index.php/en/mnutopics/chikv-en/550-chikv-weekly-en.html). Clinical infection leads to a rapid onset, self-limiting, febrile 
illness with highly debilitating arthritic sequelae sometimes lasting months or years after infection ${ }^{4,5}$, with mortality generally resulting from neuroinvasive manifestations or severe organ dysfunction ${ }^{6,7}$.

Immune response to early $\mathrm{CHIKV}$ infection has been characterized with respect to natural killer (NK) cells, known to mediate the innate response against viral infection ${ }^{8}$. Clonal expansion of NK populations was highly correlated with viral titer in endemically-infected human populations ${ }^{9}$. NK cells are not necessary for protection from CHIKV infection ${ }^{10}$, instead displaying markedly altered expression of innate immune modulators such as IFN- $\square^{9,11}$, and may instead play a role in development of chronic arthritis arising from infection ${ }^{12,13}$.

A functional Type I Interferon response, important in the activation of monocytes and macrophages $^{14}$, is necessary for protective immunity to CHIKV infection ${ }^{15,16}$. Monocytes and macrophages provide antiviral activities during infection ${ }^{17,18}$, as well as immunoregulatory functions to resolve inflammation ${ }^{19}$. In addition to their protective role during infection, monocytes and macrophages are likely to play a role in joint damage in CHIKV-induced arthritis, appearing in affected tissue along with mononuclear lymphocytes ${ }^{13,20}$. CHIKV actively replicates in monocytes/macrophages ${ }^{21}$, and persists within the cells long-term, with macrophages being major viral reservoirs ${ }^{22}$.

Dendritic cells (DCs) are an important cell type for early response to CHIKV infection ${ }^{13}$, with abrogated DC responses associated with increased disease severity ${ }^{23}$. Myeloid DCs (mDCs) exhibit a lowered number and delayed response in aged Rhesus macaques, coinciding with higher viral titers during infection ${ }^{24}$. Plasmacytoid dendritic cells (pDCs) also control CHIKV infection through Type I Interferon responses post CHIKV-infected cell contact, though NF-KB responses are excluded, thus potentially avoiding damage associated with other innate inflammatory responses ${ }^{25}$. This points to DCs as important players in the early control of infection.

In this study, we investigated innate immune responses by myeloid lineage cells, which include monocytes, macrophages, and dendritic cells in acute CHIKV infection that respond before the onset of 
adaptive immunity. We examined the phenotypic patterns and kinetics of monocytes, mDCs and pDCs during early Chikungunya infection in cynomolgus macaques. The data presented here will expand on current knowledge surrounding the innate immune responses to early CHIKV infection, which contribute to both pathogen clearance and long-term pathological consequence arising from this disease.

\section{Materials and Methods}

\section{Animals}

Age-matched cynomolgus macaques (Macaca fascicularis) weighing 3-6 kg, free of simian immunodeficiency virus (SIV), simian type D retrovirus (SRV), simian T-lymphotropic virus (STLV), and alphavirus antibodies against western (WEEV), Venezuelan (VEEV), and eastern equine encephalitis virus (EEEV), Sindbis virus (SINV), Semliki Forest virus (SFV), and CHIKV (assayed by hemagglutination inhibition) were used. The study was approved by the Institutional Animal Care and Use Committee at Tulane University, and all animals were handled in accordance with guidance from the American Association for Accreditation of Laboratory Animal Care.

\section{Viral Challenge}

Anesthetized macaques were challenged with a single subcutaneous inoculation in the upper deltoid with wild-type CHIKV-LR (5.0 $\log _{10}$ PFU in a volume of $\left.100 \mu \mathrm{l}\right)$. Blood was collected on days 1$3,6,9,13$, and 35 after challenge, when the experiment was terminated and necropsies were performed. Tissues were placed in $10 \%$ zinc-formalin for histopathological analysis: brain, lung, heart, spleen, liver, kidney, mesenteric lymph node, bronchial lymph node, jejunum, colon, testes, muscle, skin, bone marrow, eye, and knee (or finger) joint. Some tissues were also frozen for viral titration by plaque assay: axillary, bronchial, and inguinal lymph nodes. 


\section{BrdU inoculation}

BrdU was dissolved in PBS at $30 \mathrm{mg} / \mathrm{mL}$ and inoculated intravenously at $60 \mathrm{mg} / \mathrm{kg}$ body weight two days post-challenge. EDTA blood was collected at 1, 4, 7 and 11 days post BrdU administration.

\section{Flow cytometric analysis}

EDTA blood was stained for flow cytometric analysis. The following monoclonal antibodies (mAbs) were used in this study: anti-BrdU-FITC (3D4, BD Biosciences, San Jose, CA), CD45-PE (MB46D6, Miltenyi Biotec, San Jose, CA), CD20-ECD (B9E9, Beckman Coulter, Indianapolis, IN), CD123PerCP Cy5.5 (BD), HLA-DR-PE-Cy7 (L243 BD Biosciences), CD11c-APC (BD), CD3-Alexa Fluor 700 (SP34-2, BD Biosciences), CD16-APC-H7 (3G8, BD Biosciences), CD14-Pacific Blue (M5E2, BD Biosciences), CD8-AmCyan (SK1, BD Biosciences), CD4-PerCP-Cy5.5 (L200, BD Biosciences), NKG2a-APC (Z199, Beckman Coulter). Blood was first stained with surface antibodies for 20 min. After lysing red blood cells with $1 \times$ FACS lysing solution (BD Biosciences), cells were permeabilized with Cytofix/Cytoperm (BD Biosciences) for 20 min, Permwash buffer (BD Pharmingen) supplemented with $10 \%$ dimethyl sulfoxide for $10 \mathrm{~min}$, and Cytofix/Cytoperm for $5 \mathrm{~min}$. The cells were incubated with DNase for $1 \mathrm{~h}$ at $37{ }^{\circ} \mathrm{C}$, incubated with anti-BrdU antibody for $20 \mathrm{~min}$, and fixed with $1 \%$ paraformaldehyde (Electron Microscopy Systems). Cells were acquired with LSR II (BD Biosciences) and analyzed using FlowJo software (TreeStar Inc).

\section{Complete blood counts and absolute counts of each cell population}

The complete blood counts were analyzed on an ADVIA 120 Hematology System (Bayer Diagnostics) The absolute counts of CD14+ monocytes, mDC, pDC, CD4+ T cells, CD8+ T cells, B cells, and NK 
cells in blood were calculated from CBC data and flow cytometric analysis for each cell population.

\section{Statistical analysis}

The statistical analysis was performed using Graphpad Prism software (GraphPad, San Diego, CA). Analysis was performed on post-exposure data defined as significant by the established detection thresholds for fever intensity, fever (hyperthermia) hours, and hypothermia hours. Fever intensity, indicated as the maximum change in temperature, fever hours, and hypothermia hours among treatment groups, was compared using the Kruskal-Wallis test. When the results of Kruskal-Wallis test indicated a significant difference at the $P<0.05$ level between the groups examined, Tukey's multiple comparison test was then used to identify groups that differed at the $P<0.05$ significance level.

\section{Results}

\section{Viral kinetics and gating strategy}

Following challenge, virus levels were examined each day via plaque assay. Blood levels of CHIKV fell rapidly after initial peaks of $5.0 \log _{10}$ at day 2 post challenge. By day 3 post challenge, viremia was absent in five out of seven animals (Table 1).

Cells were subdivided based on gating of cell markers. Monocytes, $\mathrm{mDC}$ and $\mathrm{pDC}$ were identified as CD45+CD3-CD20-CD8-CD14+ cells, CD45+HLA-DR+Lin (CD3, CD20, CD8, and CD14)-CD11c+ cells, and CD45+HLA-DR+Lin-CD123+ cells, respectively. CD14 and CD16 expression pattern was used for subdividing monocyte population (Figure 1B).

\section{Turnover of myeloid lineage cells during acute infection}


We examined kinetics of monocyte, $\mathrm{mDC}$ and $\mathrm{pDC}$ turnover by using in vivo BrdU labeling and flow cytometric analysis. BrdU was administered into CHIKV infected animals two days post challenge and blood was collected 1, 4, 7, and 11 days later. We compared the kinetics of the percentages of BrdU+ cells in monocytes, $\mathrm{mDC}$ and $\mathrm{pDC}$ between uninfected macaques and acutely CHIKV-infected macaques (Figure 2). Peripheral blood was collected at indicated time points (1 to 11 days) after BrdU administration and the appearance of BrdU+ cells was investigated in each cell population. Homogeneous kinetics of BrdU+ cells were observed in monocytes, $\mathrm{mDC}$ and $\mathrm{pDC}$ obtained from 8 uninfected macaques (Figure 2A). In contrast, the peaks of BrdU+ cells in all populations shifted to earlier time points in CHIKV-infected macaques, though this effect was only seen in two individuals for the monocyte population (Fig. 2A).

We analyzed the significance of increased cell turnover for monocytes, $\mathrm{mDC}$, and $\mathrm{pDC}$ using the data from 24h, 4 days and 24h after BrdU injection, respectively. BrdU+ monocytes and mDCs in CHIKV-infected macaques were significantly higher than that from uninfected animals (Fig. 2B).

\section{Absolute cell counts of monocytes and DCs in acute CHIKV infection}

Consistent with the Brd-based findings, monocyte counts decreased rapidly after challenge, but rebound with an increase 6 days after infection (Figure 3A). Dramatic changes were observed in mDC and pDC counts (Figure 3B and C, respectively), with mDCs largely disappearing from the blood immediately after infection, with blood pDC massively increasing at the same time.

\section{Phenotypic changes in monocytes from CHIKV-infected macaques}

CD16 expression was up-regulated within 1 day after CHIKV infection and returned to the normal level roughly one week after infection (Figure 4A). Although we couldn't obtain the blood 
samples at 2 days after infection in the first experimental group, 2 days post-CHIKV challenge was the peak of CD16 expression (Fig. 4B). CD14 expression was significantly higher 3 days post challenge, as compared to pre-challenge (Fig. 4C).

Furthermore, we determined that the HLA-DR expression on monocytes decreased in acute CHIKV infection (Figure 5). Although DC populations were HLA-DR-positive, the down-regulation of HLA-DR was not observed in either mDC or pDC (Fig. 5A). HLA-DR-negative or low monocytes appeared in 1 day after infection and returned to the uninfected level within 1 week after infection in most animals (Figure 4B). The majority of HLA-DR-down-regulated monocytes were CD16-negative (data not shown). There was no difference in the cell turnover between HLA-DR low/neg monocytes and HLA-DR hi monocytes (data not shown).

\section{Cell turnover and absolute counts of T cell, B cell and NK cell in acute CHIK infection}

We examined the cell turnover and the absolute counts of the cell population related to adaptive immunity such as CD4+ T cells, CD8+ T cells, and B cells in blood. We also analyzed those for NK cells, since it has been reported that a phenotype change and a clonal expansion were observed in NK cells in acutely CHIKV-infected humans ${ }^{9}$. CD4+ T cells, CD8+ T cells, B cells, and NK cells temporarily decreased in a similar fashion after infection (Fig. 6).

Although the kinetics of the cell counts were similar, there were differences in the turnover among the cell populations. The cell turnover rates of CD4+ T cells were significantly decreased in acute infection (Fig. 7A), with CD8+ T cells also trending toward significant decreases (Fig. 7B). Cell turnover rates of B cells and NK cells were not significantly altered after infection (Figure 7C and D). 


\section{Discussion}

The work presented here demonstrates myeloid lineage cell alteration in number, turnover and phenotype during infection with CHIKV. We demonstrated increased turnover of monocytes and mDCs early during CHIKV infection, which did not extend to pDC cells (Fig. 2). These changes tracked well with cell counts showing an early decrease of monocytes and mDCs, with an increase over time in all cell types, but an early increase of pDCs (Fig. 3). pDCs are known as type I IFN-producing cells that rapidly secrete massive amounts of type I IFN after activation via TLR7 and/or TLR9 by sensing ssRNA or CpG motifs in DNA derived from viral and bacterial genomes ${ }^{26,27}$. Therefore, extremely high CHIKV viral loads might result in an increased number of $\mathrm{pDC}$, activation of $\mathrm{pDC}$ and induction of type I IFN by pDC. The early drop in circulating mDCs may be from increased tissue distribution, or an increase in apoptosis. Both are seen in SIV-infected macaques, along with a large drop in $\mathrm{mDC}$ number early during infection in one species of macaque, coinciding with more pathogenic infection; only a transient depletion is characterized during less severe disease ${ }^{28}$. We have found that the cell turnover in monocytes during SIV infection is highly correlated with progression to $\operatorname{AIDS}^{29}$, indicating monocyte/macrophage dynamics can play a large role in disease progression.

These myeloid lineage cell changes were accompanied by alterations in the phenotype of monocytes, with CD16 expression peaking at 2 days post-challenge, and CD14 expression peaking at day 3 (Fig. 4). CD16 is a marker of activation in monocytes, and corresponds to increases in proinflammatory cytokine secretion, which are seen in early CHIKV infection ${ }^{30,31}$. A considerable increase in the number of CD14+CD16+ monocytes had been described for a variety of systemic, infectious agents in humans, including hemolytic uremic syndrome ${ }^{32}$, bacterial sepsis ${ }^{33}, \mathrm{HIV}^{34}$, and experimental SIV infections in nonhuman primates ${ }^{35}$.

Monocytes expressing HLA-DR significantly dropped early during CHIKV infection, with a rebound seen by one week post challenge. This effect was not seen in dendritic cells (Fig. 5). Decreased 
HLA-DR expression is a hallmark of deactivated monocytes in patients with systemic inflammation, and this immune status is often referred to as compensatory anti-inflammatory response syndrome ${ }^{36}$. In in vitro studies, IL-10, an anti-inflammatory cytokine, induces HLA-DR down-regulation on monocytes ${ }^{37}$. Plasma IL-10 levels increased with increased induction of pro-inflammatory cytokines in acutely CHIKVinfected humans ${ }^{38}$.

We observed a temporary decrease in CD4+ and CD8+ T cells, as well as B cells and NK cells early during CHIKV infection (Fig. 6). This result corresponds to the lymphocytopenia observed in prior nonhuman primate models ${ }^{22}$, as well as in humans ${ }^{39}$. Turnover rates were altered during acute infection, with a decrease in rates of T cells, but no change in B cells or NK cells (Fig. 7). This suggests that proliferating CD4+ T cells and CD8+ T cells might preferentially relocate from the blood circulation to the effector sites.

These findings supplement previous work characterizing macrophages as virus reservoirs in cynomolgus macaques $^{22}$. More detail regarding kinetics and phenotype of immune cells during infection with CHIKV can aid in the development of therapeutics, as well as provide more detail as to the relevance of non-human primate models to human infection.

\section{Conclusion}

The early responses to chikungunya infection in the cynomolgus macaque are further examined here. Our findings suggest that this viral infection affects the production and turnover of myeloid lineage cells, especially monocytes and dendritic cells, as evidenced by their dramatic change in the absolute number and phenotypes early after infection. Future work should further elucidate how these phenotypic changes and turnover contribute to pathologic mechanisms of acute CHIKV infection, as well as its pathologic sequalae. 


\section{Author Disclosure Statement}

No competing financial interests exist.

\section{Acknowledgements}

This work was supported by a grant from the National Institute of Allergy and Infectious Disease

(NIAID) through the Western Regional Center of Excellence for Biodefense and Emerging Infectious

Disease Research, National Institutes of Health (NIH) grant U54 AIO57156. This work was also supported in part by NIH/NCRR grant number P51 RR000164.

\section{References}

1 Weaver, S. C. \& Lecuit, M. Chikungunya virus and the global spread of a mosquitoborne disease. $N$ Engl J Med 372, 1231-1239, doi:10.1056/NEJMra1406035 (2015).

2 Petitdemange, C., Wauquier, N. \& Vieillard, V. Control of immunopathology during chikungunya virus infection. J Allergy Clin Immunol 135, 846-855, doi:10.1016/j.jaci.2015.01.039 (2015).

3 Leparc-Goffart, I., Nougairede, A., Cassadou, S., Prat, C. \& de Lamballerie, X. Chikungunya in the Americas. The Lancet 383, doi:10.1016/s0140-6736(14)60185-9 (2014).

4 Schilte, C. et al. Chikungunya virus-associated long-term arthralgia: a 36-month prospective longitudinal study. PLoS Negl Trop Dis 7, e2137, doi:10.1371/journal.pntd.0002137 (2013).

5 Marimoutou, C., Vivier, E., Oliver, M., Boutin, J. P. \& Simon, F. Morbidity and impaired quality of life 30 months after chikungunya infection: comparative cohort of infected and uninfected French military policemen in Reunion Island. Medicine (Baltimore) 91, 212219, doi:10.1097/MD.0b013e318260b604 (2012).

6 Gerardin, P. et al. Chikungunya virus-associated encephalitis: A cohort study on La Reunion Island, 2005-2009. Neurology 86, 94-102, doi:10.1212/WNL.0000000000002234 (2016). 
7 Economopoulou, A. et al. Atypical Chikungunya virus infections: clinical manifestations, mortality and risk factors for severe disease during the 2005-2006 outbreak on Reunion. Epidemiol Infect 137, 534-541, doi:10.1017/S0950268808001167 (2009).

8 Jost, S. \& Altfeld, M. Control of human viral infections by natural killer cells. Annu Rev Immunol 31, 163-194, doi:10.1146/annurev-immunol-032712-100001 (2013).

9 Petitdemange, C. et al. Unconventional repertoire profile is imprinted during acute chikungunya infection for natural killer cells polarization toward cytotoxicity. PLoS Pathog 7, e1002268, doi:10.1371/journal.ppat.1002268 (2011).

10 Poo, Y. S. et al. Multiple immune factors are involved in controlling acute and chronic chikungunya virus infection. PLoS Negl Trop Dis 8, e3354, doi:10.1371/journal.pntd.0003354 (2014).

11 Thanapati, S., Das, R. \& Tripathy, A. S. Phenotypic and functional analyses of NK and NKT-like populations during the early stages of chikungunya infection. Front Microbiol 6, 895, doi:10.3389/fmicb.2015.00895 (2015).

12 Miner, J. J. et al. Chikungunya viral arthritis in the United States: a mimic of seronegative rheumatoid arthritis. Arthritis Rheumatol 67, 1214-1220, doi:10.1002/art.39027 (2015).

13 Hoarau, J. J. et al. Persistent chronic inflammation and infection by Chikungunya arthritogenic alphavirus in spite of a robust host immune response. J Immunol 184, 59145927, doi:10.4049/jimmunol.0900255 (2010).

14 Suhrbier, A. Rheumatic manifestations of chikungunya: emerging concepts and interventions. Nat Rev Rheumatol 15, 597-611, doi:10.1038/s41584-019-0276-9 (2019).

15 Carpentier, K. S. \& Morrison, T. E. Innate immune control of alphavirus infection. Curr Opin Virol 28, 53-60, doi:10.1016/j.coviro.2017.11.006 (2018).

16 Rudd, P. A. et al. Interferon response factors 3 and 7 protect against Chikungunya virus hemorrhagic fever and shock. J Virol 86, 9888-9898, doi:10.1128/JVI.00956-12 (2012).

17 Haist, K. C., Burrack, K. S., Davenport, B. J. \& Morrison, T. E. Inflammatory monocytes mediate control of acute alphavirus infection in mice. PLoS Pathog 13, e1006748, doi:10.1371/journal.ppat.1006748 (2017).

18 Fox, J. M. et al. Optimal therapeutic activity of monoclonal antibodies against chikungunya virus requires Fc-FcgammaR interaction on monocytes. Sci Immunol 4, doi:10.1126/sciimmunol.aav5062 (2019).

19 Ikeda, N. et al. Emergence of immunoregulatory Ym1(+)Ly6C(hi) monocytes during recovery phase of tissue injury. Sci Immunol 3, doi:10.1126/sciimmunol.aat0207 (2018).

20 Gardner, J. et al. Chikungunya virus arthritis in adult wild-type mice. $J$ Virol 84, 80218032, doi:10.1128/JVI.02603-09 (2010).

21 Felipe, V. L. J., Paula, A. V. \& Silvio, U. I. Chikungunya virus infection induces differential inflammatory and antiviral responses in human monocytes and monocytederived macrophages. Acta Trop 211, 105619, doi:10.1016/j.actatropica.2020.105619 (2020).

22 Labadie, K. et al. Chikungunya disease in nonhuman primates involves long-term viral persistence in macrophages. J Clin Invest 120, 894-906, doi:10.1172/JCI40104 (2010).

23 Long, K. M. et al. Dendritic cell immunoreceptor regulates Chikungunya virus pathogenesis in mice. J Virol 87, 5697-5706, doi:10.1128/JVI.01611-12 (2013). 
24 Messaoudi, I. et al. Chikungunya virus infection results in higher and persistent viral replication in aged rhesus macaques due to defects in anti-viral immunity. PLoS Negl Trop Dis 7, e2343, doi:10.1371/journal.pntd.0002343 (2013).

25 Webster, B. et al. Plasmacytoid dendritic cells control dengue and Chikungunya virus infections via IRF7-regulated interferon responses. Elife 7, doi:10.7554/eLife.34273 (2018).

26 Swiecki, M., Gilfillan, S., Vermi, W., Wang, Y. \& Colonna, M. Plasmacytoid dendritic cell ablation impacts early interferon responses and antiviral NK and CD8(+) T cell accrual. Immunity 33, 955-966, doi:10.1016/j.immuni.2010.11.020 (2010).

27 Swiecki, M. \& Colonna, M. The multifaceted biology of plasmacytoid dendritic cells. Nat Rev Immunol 15, 471-485, doi:10.1038/nri3865 (2015).

28 Wijewardana, V. et al. Kinetics of myeloid dendritic cell trafficking and activation: impact on progressive, nonprogressive and controlled SIV infections. PLoS Pathog 9 , e1003600, doi:10.1371/journal.ppat.1003600 (2013).

29 Hasegawa, A. et al. The level of monocyte turnover predicts disease progression in the macaque model of AIDS. Blood 114, 2917-2925, doi:10.1182/blood-2009-02-204263 (2009).

30 Vishnyakova, P. et al. The response of two polar monocyte subsets to inflammation. Biomed Pharmacother 139, 111614, doi:10.1016/j.biopha.2021.111614 (2021).

31 Ferreira, A. S., Baldoni, N. R., Cardoso, C. S. \& Oliveira, C. D. L. Biomarkers of severity and chronification in chikungunya fever: a systematic review and meta-analysis. Rev Inst Med Trop Sao Paulo 63, e16, doi:10.1590/S1678-9946202163016 (2021).

32 Fernandez, G. C. et al. Cytokine production is altered in monocytes from children with hemolytic uremic syndrome. J Clin Immunol 32, 622-631, doi:10.1007/s10875-011-9646z (2012).

33 Fingerle, G. et al. The novel subset of CD14+/CD16+ blood monocytes is expanded in sepsis patients. Blood 82, 3170-3176, doi:10.1182/blood.V82.10.3170.3170 (1993).

34 Han, J. et al. CD14(high)CD16(+) rather than CD14(low)CD16(+) monocytes correlate with disease progression in chronic HIV-infected patients. J Acquir Immune Defic Syndr 52, 553-559, doi:10.1097/qai.0b013e3181c1d4fe (2009).

35 Kim, W. K. et al. Monocyte heterogeneity underlying phenotypic changes in monocytes according to SIV disease stage. J Leukoc Biol 87, 557-567, doi:10.1189/jlb.0209082 (2010).

36 Frazier, W. J. \& Hall, M. W. Immunoparalysis and adverse outcomes from critical illness. Pediatr Clin North Am 55, 647-668, xi, doi:10.1016/j.pcl.2008.02.009 (2008).

37 Xiu, B. et al. IL-10 induces the development of immunosuppressive CD14(+)HLADR(low/-) monocytes in B-cell non-Hodgkin lymphoma. Blood Cancer J 5, e328, doi:10.1038/bcj.2015.56 (2015).

38 Venugopalan, A., Ghorpade, R. P. \& Chopra, A. Cytokines in acute chikungunya. PLoS One 9, e111305, doi:10.1371/journal.pone.0111305 (2014).

39 Borgherini, G. et al. Outbreak of chikungunya on Reunion Island: early clinical and laboratory features in 157 adult patients. Clin Infect Dis 44, 1401-1407, doi:10.1086/517537 (2007). 


\section{Viremia $\left(\log _{10} \mathrm{PFU} / \mathrm{ml}\right)$}

\begin{tabular}{lccc}
\cline { 2 - 4 } Animal no. & Day 1 & Day 2 & Day 3 \\
& & & \\
\hline HV78 & 4.3 & 5.0 & $<1.0$ \\
HV82 & 3.6 & 5.0 & $<1.0$ \\
HV84 & 3.3 & 5.0 & $<1.0$ \\
IV10 & 4.6 & 5.0 & 3.3 \\
IV11 & 4.0 & 4.6 & $<1.0$ \\
IV16 & 2.8 & 4.3 & $<1.0$ \\
IT87 & 5.2 & 5.3 & 3.5 \\
\hline
\end{tabular}

Table 1. Viremia in cynomolgus macaques after challenge with CHIKV 


\section{A}
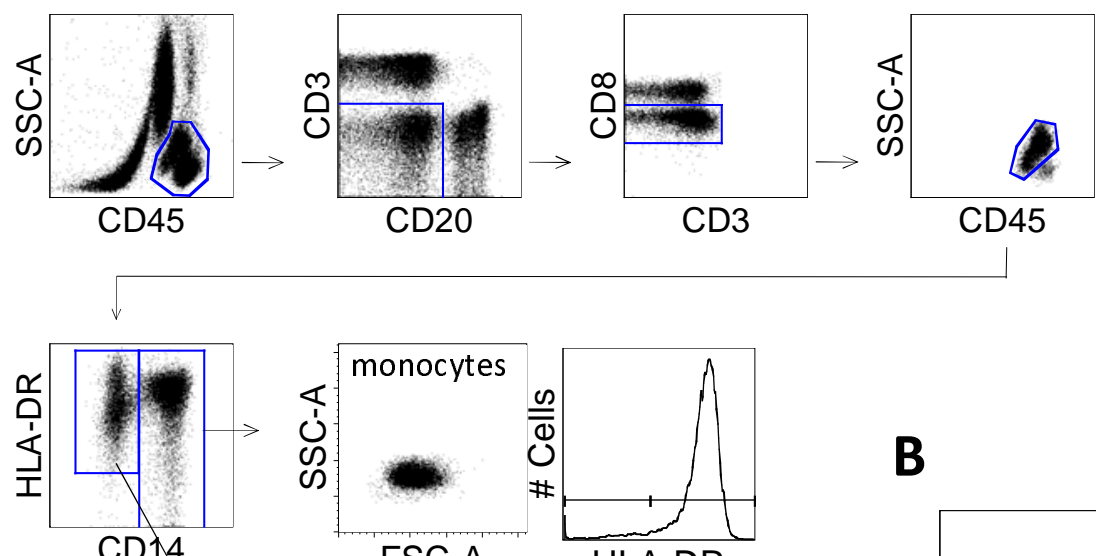

$\operatorname{CD} 14$
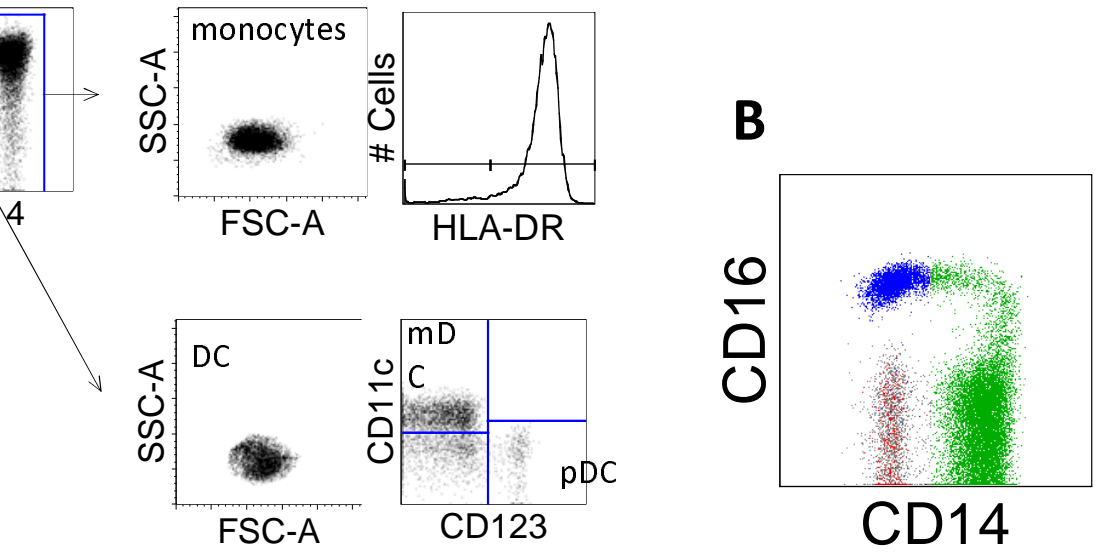

Figure 1. Gating strategy and phenotypes of myeloid lineage cells in peripheral blood from naive cynomolgus macaques. (A) Peripheral blood from naive cynomolgus macaques was analyzed by flow cytometry. Myeloid lineage cells which include monocytes and $\mathrm{DC}$ were gated as $\mathrm{CD}^{4} 5^{+} \mathrm{CD} 3^{-} \mathrm{CD} 20^{-}$ CD8. CD14 positive cells and CD14 HLA-DR ${ }^{+}$were defined as monocytes and DC, respectively. DC were further divided into $\mathrm{mDC}$ and $\mathrm{pDC}$ by CD11c and CD123 expression. (B) Monocytes (green), $\mathrm{mDC}$ (blue)and pDC (red) were recognized as distinct populations on CD14 and CD16 expression. 

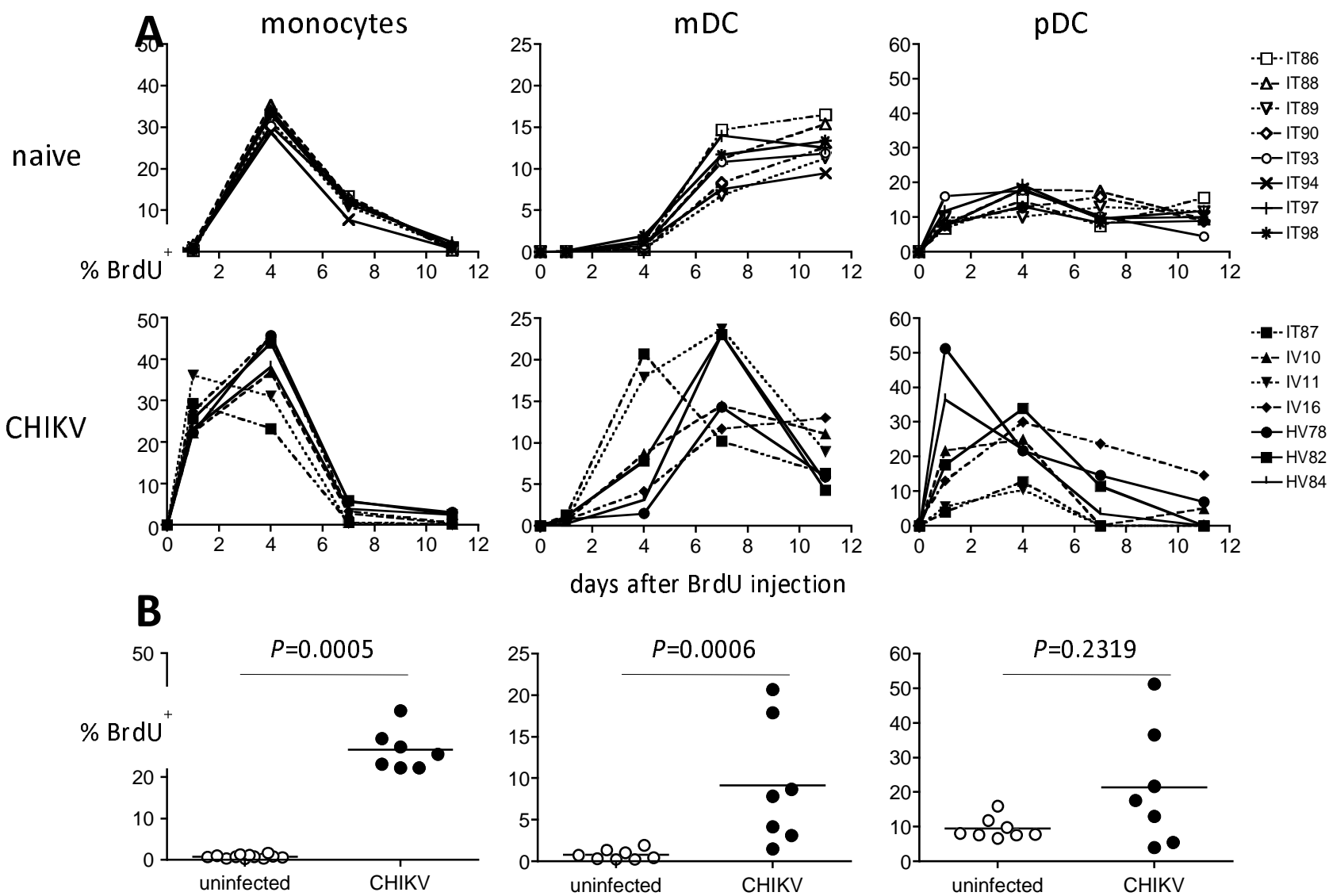

Figure 2. Kinetics of cell turnover of myeloid lineage cells in acute CHIKV infection. CHIKV-

infected macaques were administrated BrdU at 2 days postinfection. Blood was collected serially after BrdU administration and stained for flow cytometric analysis. Monocytes, $\mathrm{mDC}$, and pDC were identified as described in Figure 1. The percentages of BrdU positive cells were analyzed for each cell population. The statistical differences were analyzed by Mann-Whitney test with the data from $24 \mathrm{~h}$ (for monocytes), 4 days (for $\mathrm{mDC}$ ), and 24h (for $\mathrm{pDC}$ ) after BrdU injection. 


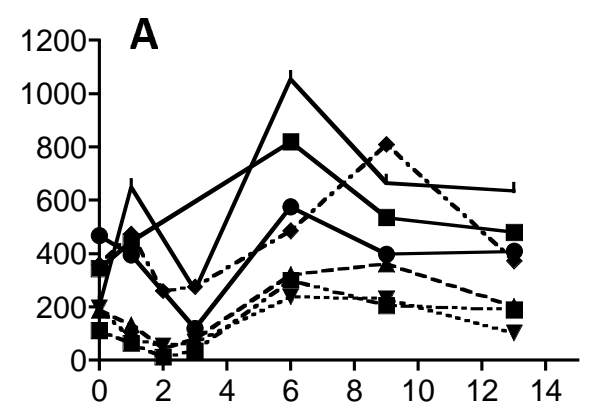

\section{\# cebto/ $\mu$ lB}
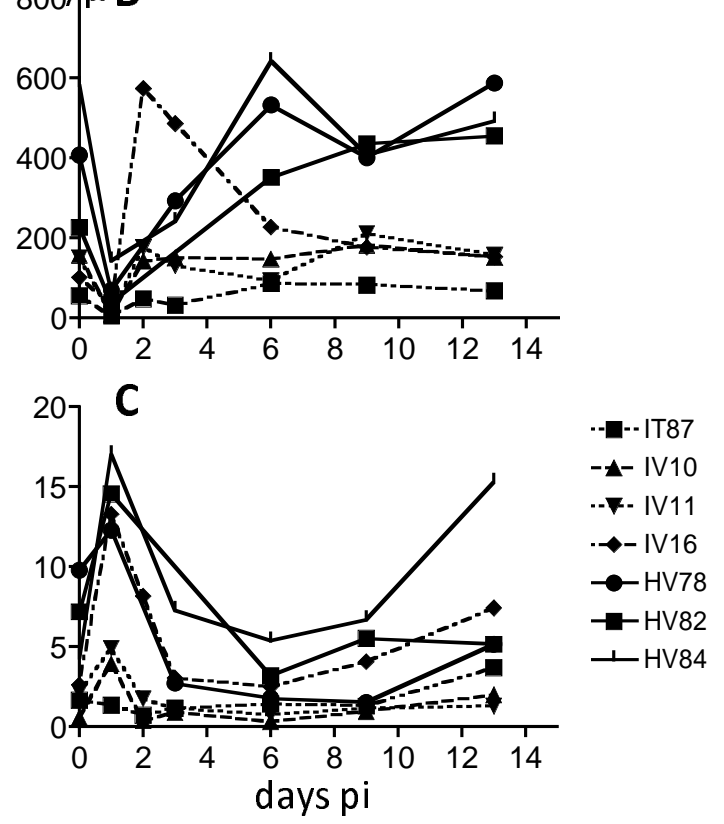

Figure 3. Absolute counts of monocytes (A), mDC (B), and pDC (C). Absolute counts of monocytes, $\mathrm{mDC}$, and $\mathrm{pDC}$ were obtained from the calculation based on flow cytometric gating and complete blood counts (CBC). 
A

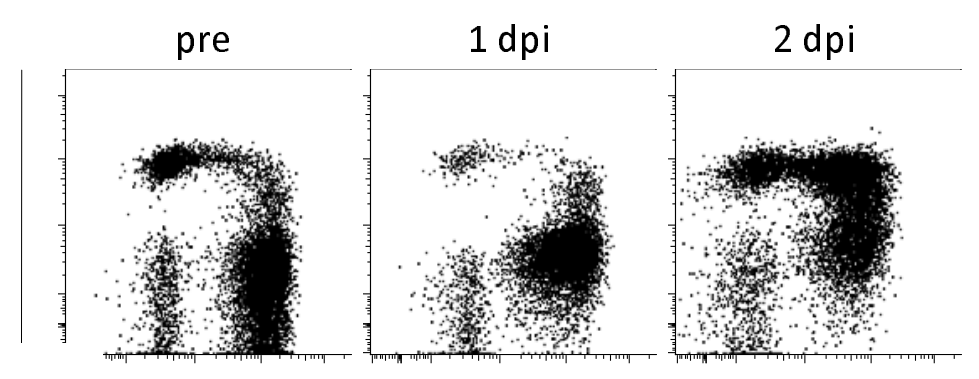

CD16

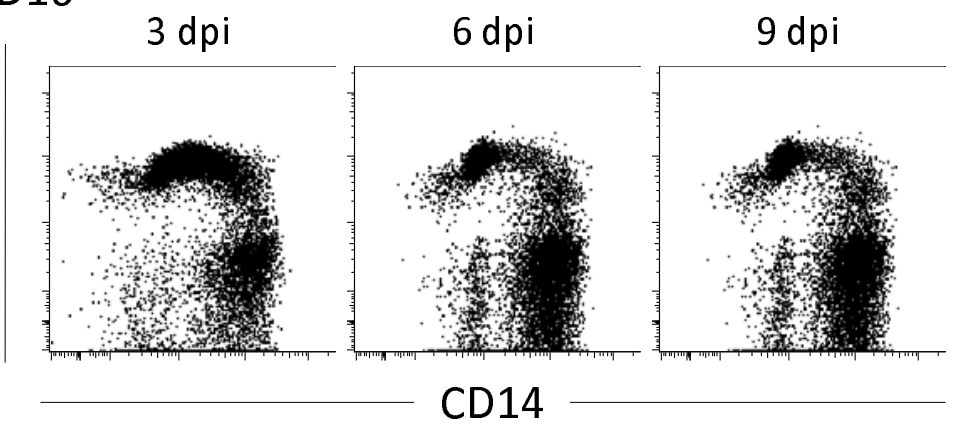

B

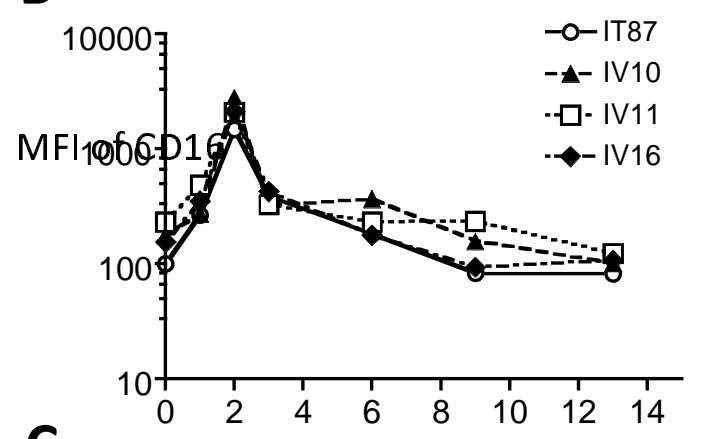

C

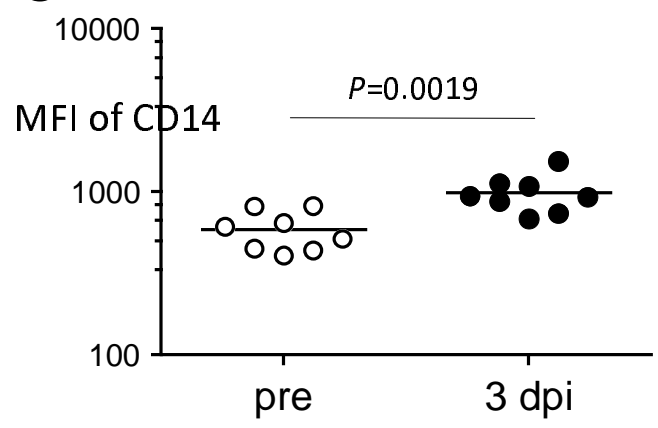

Figure 4. Altered CD14 and CD16 expression on myeloid lineage cell populations after CHIKV

infection. The $\mathrm{CD}^{4} 5^{+} \mathrm{CD} 3^{-} \mathrm{CD} 20^{-} \mathrm{CD} 8^{-}$population (as described in Figure 1) was examined for CD14 and CD16 expression by using serially collected blood after CHIKV infection. (A) Representative flow cytometric profiles from one of $7 \mathrm{CHIKV}$-infected macaques are shown. (B) Kinetic of mean fluorescence intensity of CD16 in R1 shown in (A) after CHIKV infection. (C) CD14 expression in R2 shown in (A) was compared between preinfection and 3 days after infection. The statistical difference was analyzed by Mann-Whitney test. 
A

\section{A}

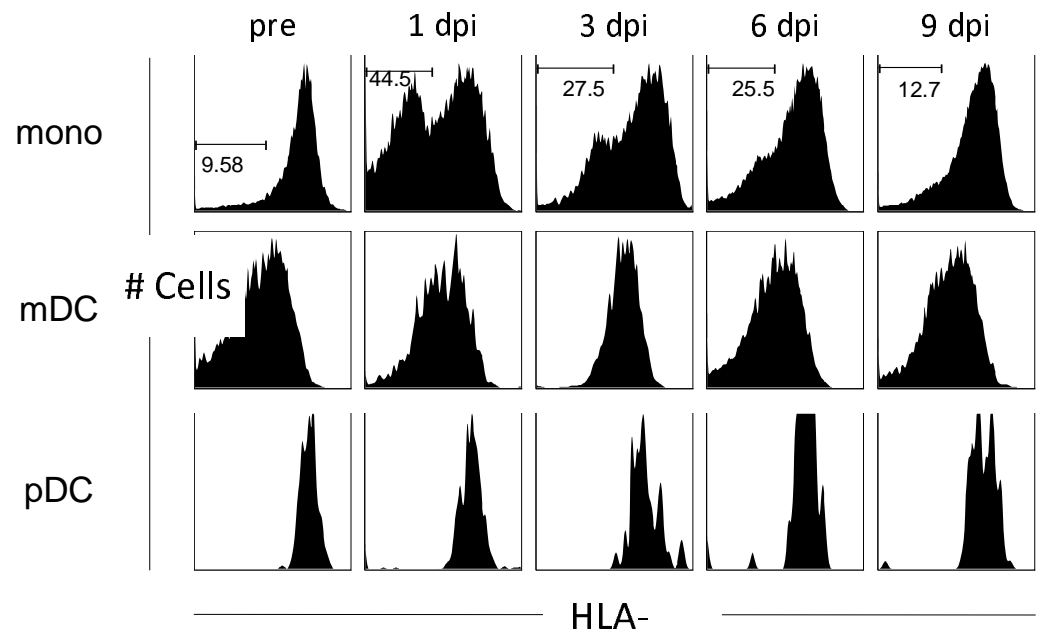

B

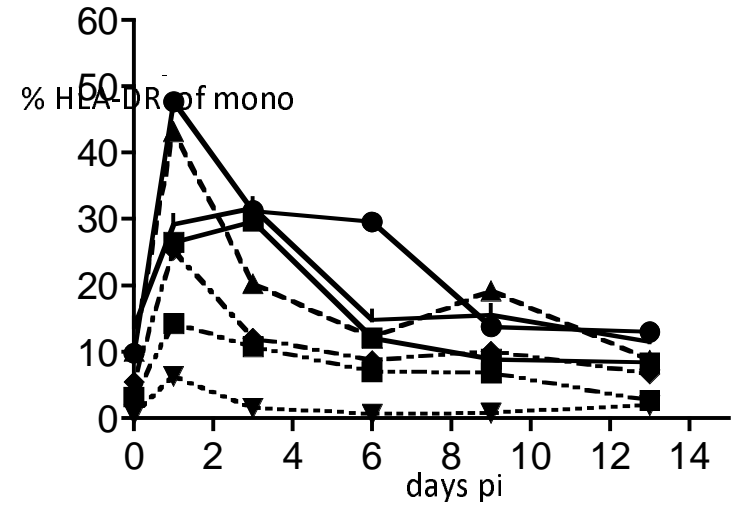

Figure 5. HLA-DR down-regulation in monocytes but not in $\mathrm{mDC}$ and $\mathrm{pDC}$ in acute CHIK V

infection. Monocytes, $\mathrm{mDC}$, and $\mathrm{pDC}$ were gated as Figure 1. The expression of HLA-DR was analyzed for each cell population. (A) Representative HLA-DR expression profiles on monocytes, mDC and pDC at the indicated days after infection are shown. (B) The percentages of HLA-DR low/negative of monocytes are plotted. 

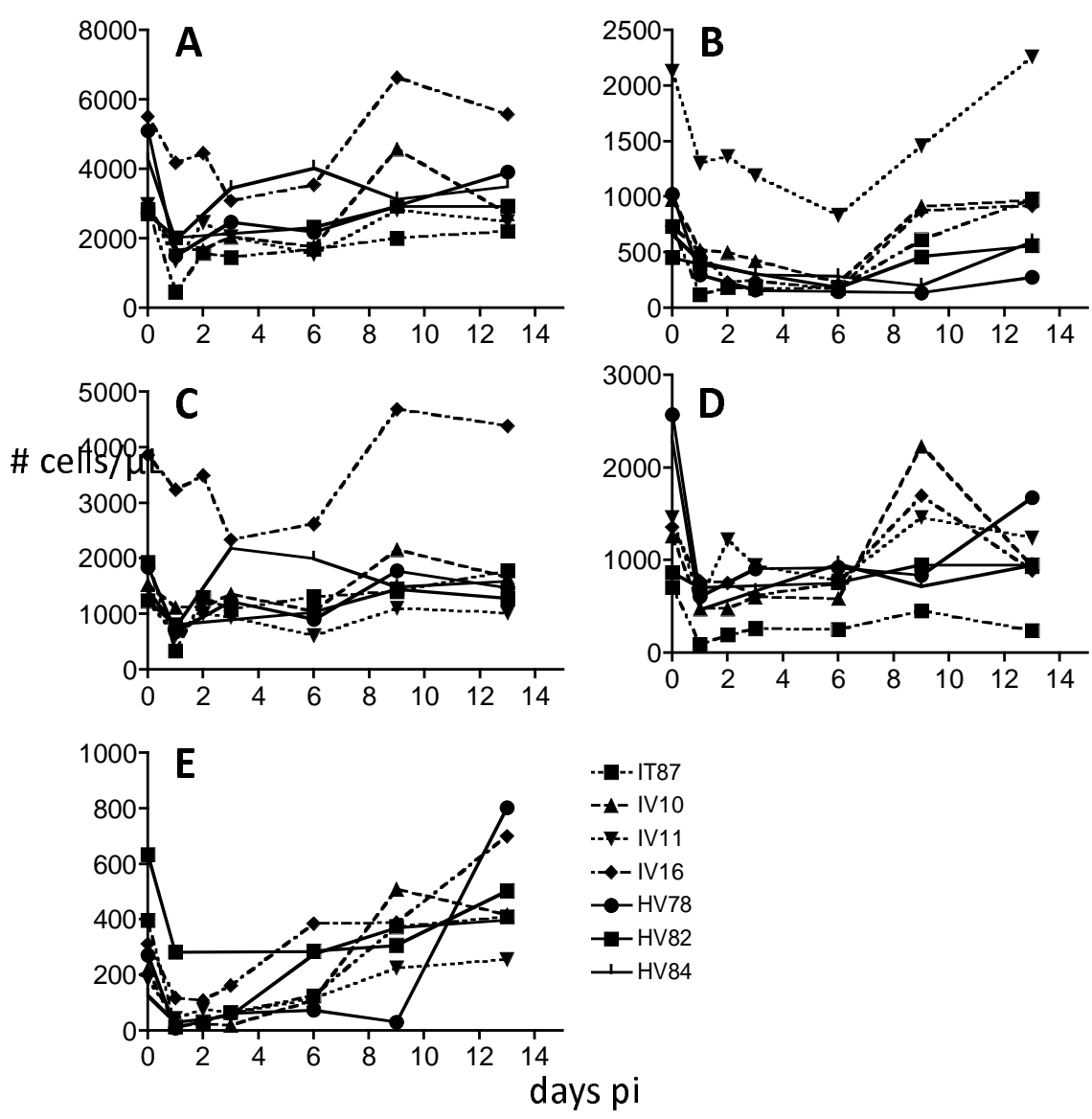

Figure 6. Absolute counts of $\mathrm{CD3}^{+} \mathrm{T}$ cells $(\mathrm{A}), \mathrm{CD20}^{+} \mathrm{B}$ cells $(\mathrm{B}), \mathrm{CD4}^{+} \mathrm{T}$ cells $(\mathrm{C}), \mathrm{CD8}^{+} \mathrm{T}$ cells $(\mathrm{D})$, and NK cells $(\mathbf{E})$. Absolute count of each lymphocyte subset was obtained from the calculation based on flow cytometric gating (as described in Figure 6) and CBC. 
A

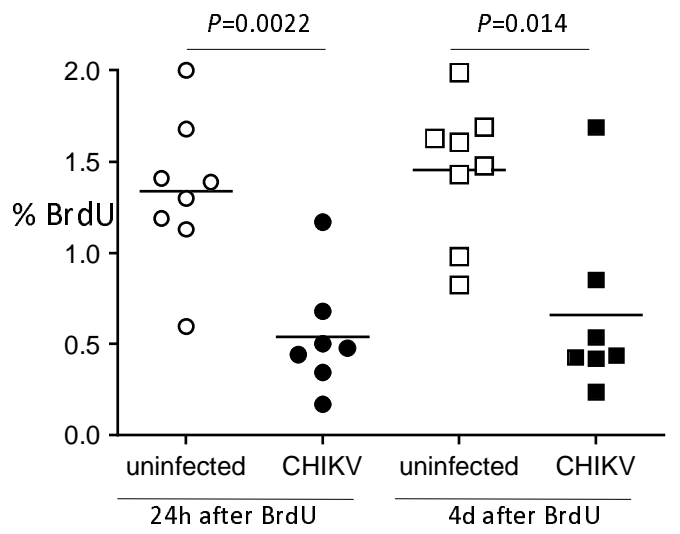

C

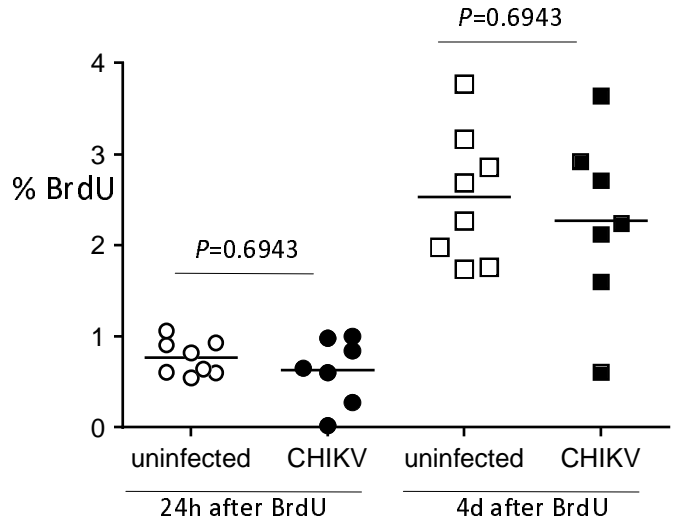

B

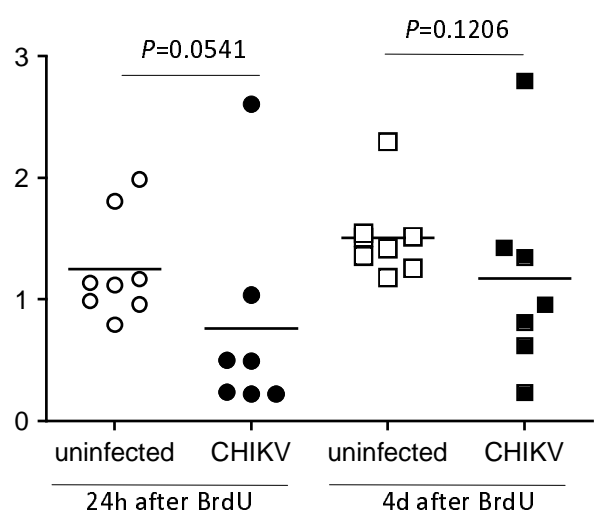

D

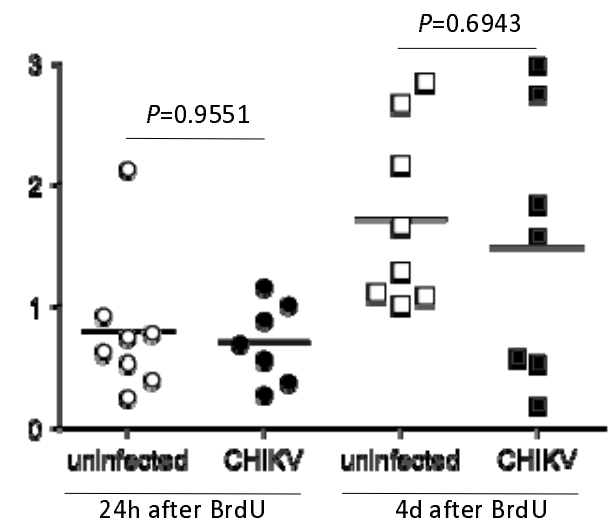

Figure 7. Lymphocyte turnover in acute CHIKV infection. CHIKV-infected macaques were administered BrdU at 2 days postinfection. Blood was collected serially after BrdU administration and stained with anti-CD3, CD4, CD8, CD20 and NKG2a, and BrdU antibodies for flow cytometric analysis. The data was compared to those form uninfected macaques. The cell turnover of $\mathrm{CD}^{+} \mathrm{T}$ cells $\left(\mathrm{CD}^{+}{ }^{+} \mathrm{CD} 20^{-} \mathrm{CD}^{-}\right), \mathrm{CD}^{+} \mathrm{T}$ cells $\left(\mathrm{CD}^{+} \mathrm{CD} 4{ }^{-} \mathrm{CD} 8^{+}\right), \mathrm{CD} 20^{+} \mathrm{B}$ cells $\left(\mathrm{CD} 3^{-} \mathrm{CD} 20^{+}\right)$, and NK cells $\left(\mathrm{CD}^{-}\right.$ $\mathrm{CD} 20^{-} \mathrm{CD} 8^{+} \mathrm{NKG}_{2} \mathrm{a}^{+}$) are shown in $\mathrm{A}, \mathrm{B}, \mathrm{C}$, and $\mathrm{D}$, respectively. The statistical differences between uninfected and CHIKV-infected groups were calculated by Mann-Whitney test. 